\title{
Springer
}

Before Refraining: Concepts for Agency

Author(s): Nuel Belnap

Source: Erkenntnis (1975-), Vol. 34, No. 2 (Mar., 1991), pp. 137-169

Published by: Springer

Stable URL: http://www.jstor.org/stable/20012334

Accessed: 28/05/2009 14:40

Your use of the JSTOR archive indicates your acceptance of JSTOR's Terms and Conditions of Use, available at http://www.jstor.org/page/info/about/policies/terms.jsp. JSTOR's Terms and Conditions of Use provides, in part, that unless you have obtained prior permission, you may not download an entire issue of a journal or multiple copies of articles, and you may use content in the JSTOR archive only for your personal, non-commercial use.

Please contact the publisher regarding any further use of this work. Publisher contact information may be obtained at http://www.jstor.org/action/showPublisher?publisherCode=springer.

Each copy of any part of a JSTOR transmission must contain the same copyright notice that appears on the screen or printed page of such transmission.

JSTOR is a not-for-profit organization founded in 1995 to build trusted digital archives for scholarship. We work with the scholarly community to preserve their work and the materials they rely upon, and to build a common research platform that promotes the discovery and use of these resources. For more information about JSTOR, please contact support@jstor.org. 


\title{
BEFORE REFRAINING: CONCEPTS FOR AGENCY
}

\begin{abstract}
A structure is described that can serve as a foundation for a semantics for a modal agentive construction such as " $\alpha$ sees to it that $Q$ " ([ $\alpha$ stit: $Q])$. The primitives are (Tree, $\leqslant$, Instant, Agent, choice). Eleven simple postulates governing this structure are set forth and motivated. Tree and $\leq$ encode a picture of branching time consisting of "moments" gathered into maximal chains called "histories." Instant imposes a timelike ordering. Agent consists of agents, and choice assigns to each agent and each moment in Tree a set of "possible choices", where each possible choice is a set of histories. All of these ingredients are referred to in the semantics suggested for $[\alpha$ stit: $Q]$. The most complex part of the discussion is the motivation for the definition of what it means for a typically non-terminating chain of moments jointly to witness the truth of $[\alpha$ sit: $Q]$ at a moment.

The paper begins with an informal account of the "Refref conjecture", which says that the only way to refrain from refraining from seeing to something is to see to it. The paper ends with a consideration of an argument of Prior's that in a certain sense contemplation and action are inconsistent.
\end{abstract}

The modal logic of agency, like any modal logic, makes it easy to think about the expressive possibilities of nesting agentive expressions. ${ }^{1}$ Thus encouraged, and building on fundamental tense logic work of Prior, Belnap and Perloff 1989 proposes a straightforward modal analysis of (an approximation to) refraining from an action. This analysis leads for instance to the "Refref conjecture", that the only way to refrain from refraining from an action is to carry out that action. This paper started out to be an account of what is known about the Refref conjecture, but in the event that story will have to be told on another occasion, for a preliminary elaboration required to talk sensibly about the conjecture has used up all the allotted time and space. The difficulty is that these ideas were only briefly and informally described in Belnap and Perloff 1989, and again in Belnap 1989; these papers instead concentrated on motivation and applications. What I therefore offer here, apart from a few informal paragraphs concerning the Refref conjecture, is the laying out in much more substantial detail of some of the fundamental ideas of the formal metaphysics and semantics of the modal logic of agency, together with a condensed application of these ideas to an argument of Prior's concerning the compossibility of contem-

Erkenntnis 34: 137-169, 1991.

(C) 1991 Kluwer Academic Publishers. Printed in the Netherlands. 
plation and action. You may well come away from a tour through the paper with the feeling that a great many pieces of machinery have been manufactured that as yet have not been given important jobs to do; if so, I hope that you will turn to the other papers mentioned above, and also accept what our admired and beloved too-soon departed colleague, Wilfrid Sellars, used to call a "promissory note".

\section{THE REFREF CONJECTURE}

The background idea is that one good way of approaching agency is via the modal locution $[\alpha$ stit: $Q$ ], to be read as " $\alpha$ sees to it that $Q$ ", where $\alpha$ is an agent and where, according to the thesis that the complement of stit should be unrestricted, $Q$ may take the place of any sentence whatsoever. ${ }^{2}$

Given the locution $[\alpha$ stit: $Q]$, it is easy to see that refraining when complemented by a non-agentive has just the form $[\alpha$ sit: $\sim Q]$; for example,

Autumn Jane refrains from becoming muddy

comes to

Autumn Jane sees to it that she does not become muddy.

Accordingly, refraining from an action has the form $[\alpha$ stit: $\sim[\alpha$ stit: $Q]]$. For example,

Autumn Jane refrains from seeing to it that she becomes muddy

comes to

Autumn Jane sees to it that it is false that she sees to it she becomes muddy.

The two forms are easy for the ear to confuse, but the reflective eye can see that advice to refrain from seeing to it that one becomes muddy is much easier to follow than advice to refrain from becoming muddy. Parents and children alike doubtless rely on the ear's confusion when they hash out the matter with each other after the dress is splattered by a passing truck.

Give a modal logician a little nesting, and more is wanted. The form 


$$
[\alpha \text { stit: } \sim[\alpha \text { stit: } \sim[\alpha \text { stit: } Q]]],
$$

which may be read as

$\alpha$ refrains from refraining from seeing to it that $Q$,

illustrates the nesting of refraining within itself. In this language the sample question noted above can be expressed as the

Refref conjecture. $[\alpha$ stit: $\sim[\alpha$ stit: $\sim[\alpha$ stit: $Q]]]$ is equivalent to $[\alpha$ stit: $Q]$.

If the Refref conjecture is true, then the only way that Autumn Jane can refrain from refraining from seeing to it that she becomes muddy is to see to it that she becomes muddy.

The conjecture is perhaps not so exciting in itself, though I confess to a certain mud-pie fondness for it. In order to think about the conjecture with precision, however, it is apparent that we shall be lost without a careful metaphysics and semantics. Neither making an effort intuitively to outline in imagination four-dimensional chunks of space time that are refrainings from refrainings, nor regaling ourselves with incident-filled dramatic stories, is going to suffice. I therefore describe the metaphysics of choice and a semantics of stit that was briefly indicated in Belnap and Perloff 1989 , in the belief that it not only contributes to the very special problem of clarifying the Refref conjecture, but is generally on a right track toward an understanding of agency. ${ }^{3}$

\section{THEORY OF BRANCHING TIME}

The metaphysics is based on branching time with agents and choices. I postulate an underlying temporal-modal-agent-choice structure $\langle$ Tree, $\leqslant$, Instant, Agent, choice $\rangle$ subject to certain constraints. These I present gradually, beginning with the theory of branching time (as it is often called).

(T1) Tree is a set whose members I call moments. I use $m$ and $w$, often with markings, as ranging over moments.

Each moment should be pictured as an instantaneous, spatially complete, really possible situation, where you may take this either as prerelativistic or as requiring some one frame of reference to make relativistic sense. ${ }^{4}$ Tree, although a set of moments, may perhaps be taken 
to represent or supervene upon a concrete entirety or world (I am of course thinking of Lewis 1986). Probably it matters whether the fundamental notion represented by "Tree" is concrete or abstract or mixed, or indeed whether the distinction itself should be taken as intramundane, but not for present purposes. What does matter is the key difference between this concept of "world" and that of Kripke 1959 (etc.), of Lewis 1986 (etc.), or of the standard four-dimensional concept derived (I suppose) from Newton by way of Einstein and Minkowski: I take the world, our world, to contain real possibilities both for what might be and for what might have been. If there are alternative worlds, then they, too, come with their real possibilities. ${ }^{5}$

(T2) Tree is partially ordered by $\leqslant$ (reflexivity, transitivity, and anti-symmetry). I use $<$ for the associated strict partial order (i.e., $\leqslant$ but not $=$ ). Picture the direction of $m_{0} \leqslant m_{1}$, which flows from past to future, as generally upwards from $m_{0}$ to $m_{1}$. In English I read $\leqslant$ and its converse with the unmarked words "earlier/later", "below/above", "lower/upper", etc., and mark by the insertion of "proper" when I intend $<$ or its converse. Moments are comparable if $\leqslant$ goes one way or the other, and a chain is a set of comparable moments; we let $c$ range over chains.

A maximal chain in Tree (no moment outside the chain is comparable to every moment inside the chain) is called a history. I use $h$, often marked, as ranging over histories, and $H$ as ranging over sets of histories. $H_{(w)}$ is the set of histories in which $w$ lies - or, to use a different geometrical phrase, the set of histories "passing through" $w$.

Perhaps this sense of history (as a set of moments) should be taken to supervene on an underlying notion of history as a concrete whole. Probably it matters whether history as a set supervenes upon history as a concrete whole, but not for present purposes. What does matter is that a history is not an entirety or world. First, our world is chocka-block with real possibilities, perhaps with chances, and certainly with actions, none of which finds a home in a single history. Second, although the phrase "our world" makes sense, "our history" does not, unless determinism be permanently true, for if it is not, our moment - the situation that is the (idealized) context of this communication - is part of many possible histories, no one of which is marked in any way as 
"ours". 6 A context of utterance can determine much, including a speaker, an auditor, a moment (whose spatial spread is given only relative to a frame of reference), a past, a focus of attention, etc., but it cannot determine a unique history of which it is a part, for (unless determinism be permanently true) there are too many histories of which it is a part. No conversational device, whether artificial or rooted in ordinary language, can overcome this difficulty, anymore than any charitable story about the use of "our child" can make it true that each couple has exactly one child.

The point is not that we cannot take the trouble to uniquely specify some history or other; maybe we can, and if so we can certainly follow Plantinga 1974 in calling it "Kronos". Nor is the point that the conversational situation cannot render some one history "salient". The point instead is that nothing can specify the (unique) history to which we and this context of utterance belongs, because (unless determinism be permanently true) no uniqueness is to be had.

Let us now look backwards.

(T3) Incomparable moments in Tree never have a common upper bound (No downward branching).

By a past or a past history (the phrases are interchangeable) I mean a nonempty upper bounded set of moments that contains every moment below any moment it contains; and I let $p$ range over pasts. Because of No downward branching, any past is a chain and thus can be extended to a history. The set of (either improper or proper) predecessors of each moment is a past. Thus, the phrase "the past" or "the past history of which the present moment is the last moment" is endowed by each context of utterance with a perfectly determinate meaning. Let $p_{(m)}$ be the past containing $m$ and all its predecessors.

In contrast, if "the future" connotes a portion of a history, then there is no sense to the phrase "the future" - unless determinism be permanently true - for the implied claim to uniqueness is false. For this reason we should distinguish between the phrases "a future" and "a future history" even though it is unnecessary to distinguish between "a past" and "a past history". By a future I mean a nonempty set of proper upper bounds of some past; each future will have the shape of a tree, and will be a subtree of Tree. By a future history I mean a nonempty maximal lower bounded chain of moments (no moment out- 
side the chain except one of its lower bounds is comparable to every moment inside the chain). ${ }^{7}$

When at a given moment Lee-Hamilton says "the past is stone, and stands forever fast", his use of the phrase "the past" safely refers to a past that is uniquely determined by the (idealized) context of utterance. Furthermore, this past is a portion of each history of which his utterance is a part: no matter the future history, that past stands forever fast.

When, however, at a certain moment Tennyson says "I dipt into the future, far as human eye could see,/Saw the Vision of the world, and all the wonder that would be", we must be careful, especially when we learn that the Vision involves a sea-battle. There is no philosophical problem, if we interpret the poet as denoting the tree of possibilities that fans out from the moment of his utterance in intricate arborescent profusion - a proper source of wonder indeed, even if our anticipation is stopped at a distant horizon. Nor if the poet is using "the future" as having its denotation relative not only to the moment of utterance but also to a history ${ }^{8}$ is there a problem, as long as we do not forget this relativization and take proper care to disambiguate Tennyson's expression accordingly, just as we would in the case of an occurrence of "the man" in ordinary discourse. If, however, by the phrase "the future" the poet intends to denote a unique future history of which his moment is a part, then his intention cannot be carried out, for (unless determinism be true from that moment as far as human eye can see) there is no such unique future history. But more of this below.

Sometimes one hears a philosopher or a physicist maunder on about distinct pasts that coalesce in a present moment, and doubtless it is good that our conceptual limits be tested. I confess, however, that I myself cannot follow these fancies. That I face alternate future histories seems to me right; that I am faced away from alternate pasts seems to me wrong. That starting with the concrete situation yesterday morning there were alternative possible ways in which yesterday might (qua time slice) have been filled seems to me right; that any of these distinct alternate pasts might have finished up in this very concrete situation seems to me wrong. One of the reasons I think this is that I do not know how to make sense out of agency on such a theory.

Some of branching time can be modeled equally well on what might be called a " $\mathrm{T} \times \mathrm{W}$ " theory (with $\mathrm{T}$ for times and $\mathrm{W}$ for worlds) after Thomason 1984, or might equally well be called a "divergence" theory after Lewis 1986, or even just a "Kamp" theory after Kamp 1979. The 
key idea is that histories (to use the present terminology) are taken as ontologically distinct each from each (no common parts), and that enough in the way of additional concepts is added to permit one to say that two histories "perfectly match up through" (Lewis) or "differ only in what is future to" (Thomason") a particular time. If, however, two histories can match before a certain time while failing to match after that time, it seems hard to deny that they can equally well match after a certain time while failing to match before that time; for this reason, such an approach to metaphysics, unlike branching time, can well encourage backwards branching babbling. Branching time, incidentally, does not deny forwards matching of distinct histories; instead what it denies is their forwards overlap. For example, it may be that one of the histories on which I turn left becomes very like one of the histories on which I turn right at some point after the heat death of the sun; why not? But "very like" is not the same as sharing particular moments (and is not even very like).

For later reference I insert a little more about pasts and histories. A history extends or is through a past just when it is a superset of that past. By $H_{(p)}$ I mean the set of all histories that extend $p$. A moment, $m$, is a minimal upper bound for a past, $p$, if $m$ is an upper bound for $p$ such that no other upper bound for $p$ is properly earlier than $m$. A past may have no minimal upper bounds, or more than one minimal upper bound, or a unique minimal upper bound; in the last case I speak of a least upper bound. I say that $p$ is complete if it contains any least upper bound that it may have. Observe that a complete past may have no minimal upper bound, or more than one. I let $p^{*}$ be the completion of $p$, that is, the smallest complete past extending $p$, so that $p$ is complete just in case $p^{*}=p$. Evidently $p_{(m)}$ is a complete past.

Every two moments have a lower bound (Historical connection); but I do not suppose that they have a greatest lower bound (Semi-lattice condition).

So every two histories share a common past: their nonempty set-theoretical intersection. When in fact there is a least upper bound, $w$, of the intersection of two histories in a common past, I call $w$ the moment of intersect of the two histories, and also say that the two histories intersection in $w$. There may also be a greatest lower bound of those moments in the two histories that are not in their overlap, which I call the moment of splitting, and I say that the two histories split at such a 
moment. When either the moment of intersection or the moment of splitting exists, they both exist, and they are identical. Because, however, the world may not be kind enough to verify the Semi-lattice condition, we cannot be sure that for each two histories there is a moment at which they split. ${ }^{10}$ (The picture of a "moment of splitting" is good when one is tracing the two histories upwards, beginning before they have split. The picture of a "moment of intersection" is good when one is tracing the two histories downwards, beginning before they have intersected.)

It is not supposed that all the moments in Tree have a common lower bound, which would imply a Big Bang. It is Historical connection that puts all moments of Tree "in suitable external relations", as Lewis 1986 says (p. 208). It gives content to exactly the sort of real possibility that I think pertinent to an understanding of stit. ${ }^{11}$

\section{THEORY OF INSTANTS}

If "histories" are a way of making a sort of vertical division of Tree, then Instant is a kind of horizontal counterpart.

Instant is a partition of Tree; that is, Instant is a set of nonempty sets of moments such that each moment in Tree belongs to exactly one member of Instant. The members of Instant are called instants; $i$, often marked, ranges over instants, and $i_{(m)}$ is the uniquely determined instant to which a moment $m$ belongs.

Instants are perhaps not fully "times" because I am not in this study relying on measures or distances, but it is intuitively correct to think of $i_{(m)}$ as the set of alternate possibilities for "filling" the time of $m$. I need instants because I think that for the sense of stit I am after, in considering whether Autumn Jane stit she was muddy at a certain moment, it is relevant to consider what else might have been at the instant inhabited by that moment. ${ }^{12}$

Next I enter a pair of conditions on Instant that are very likely too strong (too oversimplifying); my justification is that agency is already hard to understand, so that it won't hurt to try to see what it comes to in circumstances that are not altogether realistic - as long as we keep track of what we are doing so that later we can try to move closer to reality. 
(T6) Each instant intersects each history in a unique moment. I let $m_{(i, h)}$ be the moment in which instant $i$ cuts across (intersects with) history $h$.

(T7) Instants never distort historical order: given two distinct instants $i_{1}$ and $i_{2}$ and two histories $h$ and $h^{\prime}$, if the moment at which $i_{1}$ intersects $h$ is earlier than the moment at which $i_{2}$ intersects $h$, then the same relation holds between the moment at which $i_{1}$ intersects $h^{\prime}$ and the moment at which $i_{2}$ intersects $h^{\prime}$. In symbols, $m_{\left(i_{1}, h\right)}<m_{\left(i_{2}, h\right)}$ implies $m_{\left(i_{1}, h^{\prime}\right)}<m_{\left(i_{2}, h^{\prime}\right)}$.

Thus, which most persons think not so plausible, all histories have exactly the same temporal structure. Obviously we may induce a linear order on Instant by defining $i_{1} \leqslant i_{2}$ iff $m_{1} \leqslant m_{2}$ for some moment $m_{1}$ in $i_{1}$ and some moment $m_{2}$ in $i_{2}$. It follows that all histories are isomorphic with each other, and with Instant; but no assumption whatsoever is made about the order type that all histories share with each other and with Instant. For this reason the present theory of agency is immediately applicable regardless of whether we picture succession as discrete, dense, continuous, well-ordered, some mixture of these, or whatever; and regardless of whether histories are finite or infinite in one direction or the other.

In particular, I do not assume that each upper bounded subset of a history has a least upper bound in that history (No gap condition). It may, however, be so; it may be, for instance, that each history, and Instant, have the structure of the real line.

The theory of Instant is not as fundamental as that of $\leqslant$, and perhaps it is too strong, even pre-relativistically. Certainly the present assumption that all histories have isomorphic temporal orderings is stronger than comparable assumptions of Thomason 1970 or Thomason and Gupta 1980, and perhaps it should ultimately be weakened. In the meantime, while it is good to be concerned about over-simplification, the assumption can be clarifying when it comes to thinking about certain aspects of agency. Instant gives us a theory of linear "time" to play off against the theory of branching "time" based on $\leqslant$.

Together the No gap condition and the Semi-lattice condition (neither of which we assume satisfied) amount to this: every past has a least upper bound (Lub condition). For the No gap condition says that every past has at least one minimal upper bound in Tree (an absence of a 
minimal upper bound for a past would constitute a gap), and the Semilattice condition says that every past has at most one minimal upper bound in Tree (a pair of distinct minimal upper bounds for a past would violate the Semi-lattice condition).

By the completion of $\langle$ Tree, $\leqslant\rangle$ I mean the structure $\langle$ Tree*, $\leqslant *\rangle$ consisting of the set of complete pasts of Tree ordered by subset. Obviously Tree is embedded in Tree* by taking $m$ into $p_{(m)}$, but evidently Tree* also contains additional points, which I might from this perspective call quasi-moments. In some respects the theory of Tree* is nicer than that of Tree; for one thing, it clearly satisfies the Semilattice, No gap, and Lub conditions. But there is a problem lurking: it may not be possible to add a sensible Instant*. If a quasi-moment is added to Tree* because of a gap, i.e., because of a past without an minimal upper bound, then there is no problem, for the fact that all histories in Tree and Instant itself are isomorphic makes it easy to see that we can add an appropriate Instant* to Tree*. The trouble comes if a quasi-moment is added because of a failure of the Semi-lattice condition. In that case, since the Semi-lattice condition might fail where one past approaches an instant $i$ but not where another past approaches $i$, isomorphism could break down to such an extent that there would be no method of partitioning Tree* by an Instant* in such a way as not to distort order. It would be good if those who think the isomorphism condition is too strong tried to make some headway with understanding this matter; in the meantime, I do not know of a way to pass to the completion Tree* of Tree, even if I thought I had good reason to do so, but must stay with Tree itself.

\section{DETERMINISM AND ITS DENIAL}

This theory about Tree does not contain an explicit denial of determinism, but at least I can give a slightly freshened account of what determinism means.

First I need a concept. Two histories extending a past, $p$, are $u n$ divided at $p$ just in case they appear as a single line as the completion $p^{*}$ of $p$ comes to a close: that is, the two histories have in common some moment that properly follows $p^{*}$. I also say the same thing with "moment" in place of "past": two histories are undivided at a moment, $w$, if at $w$ they appear as if they were a single line. That is, not only 


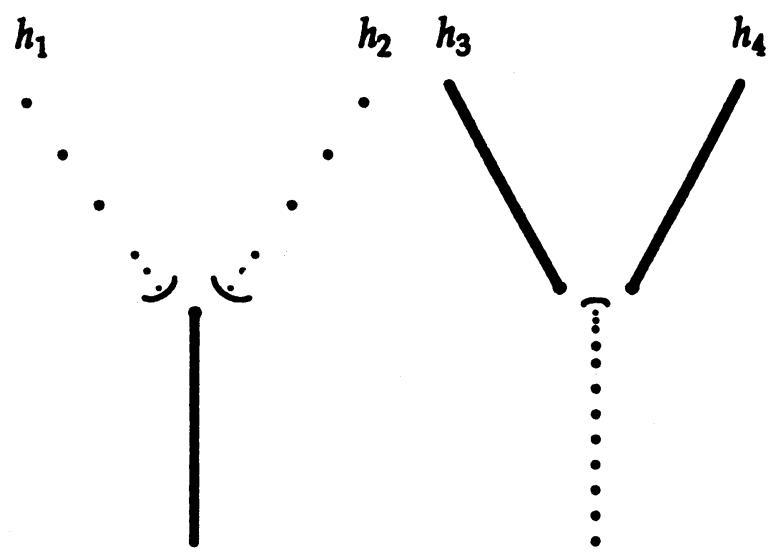

Fig. 1. Two indeterministic trees.

must they pass through $w$, but they must also share some properly later moment.

Let us say that Tree is deterministic at $p$ (or, as a variant, that the past, $p$, is deterministic) if every pair of histories through $p$ are undivided at $p$. There may be many histories through a deterministic past, $p$, but if so, they must become "many" after $p^{*}$ is past; there is no branching as $p^{*}$ itself comes to a close if $p$ is deterministic.

In this discussion I am using a past $p$ only to mark the transitional "point", and indeed given the Lub condition that we do not have, the entire discussion could have been given in terms of moments instead of pasts. (A moment, $m$, is deterministic if every pair of histories through $m$ are undivided at $m$.) Here and also elsewhere, complete pasts do work for us similar to that done by Dedekind cuts. To see the work done, here is a picture of two indeterministic trees.

The left-hand tree contains an indeterministic moment, namely, the moment of splitting of the two histories, $h_{1}$ and $h_{2}$. But the right-hand tree contains no moment of splitting at all, and therefore does not contain an indeterministic moment: whenever the pair of histories $h_{2}$ and $h_{4}$ in the right-hand tree both pass through a moment, the two histories look as if they were a single line (that is, they have in common a moment yet later than each moment through which they both pass). The common past of $h_{3}$ and $h_{4}$ is, however, indeterministic: that past is complete, and $h_{3}$ and $h_{4}$ share no moment beyond that past.

Obviously Tree may be deterministic at $p$ but not so at either earlier 
or later pasts. Equally obviously, I have not mentioned "laws" nor anything else even remotely linguistic, nor even "scientific". On the present account of determinism, one can coherently believe that our world used to be deterministic but is not so now, although it may become so once again. I go on to say that Tree itself is deterministic if it is deterministic at every past. In that case there is obviously but a single history. A determinist is someone who believes that the tree (world) of which our moment is a part is deterministic. It would appear that many philosophers believe that anyone who is not a determinist is softheaded and probably needs therapy. Others believe that anyhow all respectable philosophical theories, including theories of agency, should at least be consistent with determinism. Determinism, however, is an extremely strong theory, going far beyond determinism of the present moment. In any event, I am not a determinist (although the denial of determinism is not a postulate of this paper) and so can leave this worry to others; on the theory here offered, if anyone could ever see to anything, then determinism is false.

It could be that Tree is strongly anti-determinist in the sense that there is splitting of histories at every moment, so that $H_{(m)}=H_{\left(m^{\prime}\right)}$ implies $m=m^{\prime}$ for every $m$ and $m^{\prime}$ in Tree. It seems to me entirely possible that strong anti-determinism is true - but maybe not. Certainly it would be technically attractive to be able sometimes to think of a moment as a set of histories (those that pass through it), just as we can think of a history as a set of moments (those that it passes through), but with equal certainty, this is not a good place at which to argue from beauty to truth.

\section{THEORY OF AGENTS AND CHOICES}

I postulate that the concept of Agent is absolute in the sense of Bressan 1972 (or better, a substance sort in the sense of Gupta 1980), which in particular means that there is no fission and no fusion of agents. In this paper, however, I am not discussing questions such as de re vs. de dicto ascriptions of agency, nor even worrying about when agents come to be or pass away, so that I can get by with a simpler set-theoretical representation of Agent.

(T8) Agent is a nonempty set. I call the members of Agent agents, 
and I let lower case Greek letters, mostly $\alpha$, range over agents.

The last parameter of our temporal-modal-agent-choice structure is choice, which is to give us information as to what choices are open for each agent at each moment in Tree.

(T9) I suppose that choice is a function defined on agents and moments that yields as value for a given agent and moment a partition of all the histories passing through that moment. ${ }^{13}$

Without getting hung up on the difference between sets and functions, the following are handy uses of "choice".

- choice represents all the choice information for the entire Tree.

- $\quad$ choice $_{\alpha}$ gives all the choice information for the agent, $\alpha$; it can be thought of as a function from moments to sets of possible choices for $\alpha$.

- $\quad$ choice $^{w}$ gives all the choice information for the moment, $w$; it can be thought of as a function from agents to sets of possible choices at $w$.

- $\quad$ choice $_{\alpha}^{w}$ gives all the choice information for the agent, $\alpha$, and the moment, $w$; it can be thought of as a set of possible choices, and we call it "the set of choices possible for $\alpha$ at w'.

- $\quad \operatorname{choice}_{\alpha}^{w}(h)$ is defined only when $h$ passes through $w$, and is then the unique possible choice (a set of histories) for $\alpha$ at $w$ to which $h$ belongs. We call such a set a possible choice for $\alpha$ at $w$; it is essential to keep in mind that a possible choice is a set of histories, not a single history.

Each history in choice ${ }_{\alpha}^{w}(h)$ may be said to be choice-equivalent to $h$ for $\alpha$ at $w$. It is the intent of the postulate on choice that when agent and moment are fixed, choice-equivalence is an equivalence relation on the histories passing through the moment: reflexive, symmetric, and transitive. For example, to say that choice $\alpha_{\alpha}^{w}(h)=$ choice $_{\alpha}^{w}\left(h^{\prime}\right)$ is another way of saying that $h^{\prime}$ belongs to choice ${ }_{\alpha}^{w}(h)$.

A contrary concept is also useful: if $h$ and $h^{\prime}$ belong to different members of choice ${ }_{\alpha}^{w}$, then we say that each of these two possible choices distinguishes the two histories, and that the two histories are choice- 
distinguished for $\alpha$ by $w$. If we wish to say this in a sentence that makes " $\alpha$ " the grammatical subject, we should say that $\alpha$ has a possible choice at $w$ that distinguishes $h$ and $h^{\prime}$ - we should not say that " $\alpha$ can choose between $h$ and $h^{\prime \prime}$ !

If there is only one possible choice for $\alpha$ at $w$, it will evidently be the set of all histories passing through $w$, and I will say that it is vacuous. If there is more than one possible choice for $\alpha$ at $w$, I will call $w$ a choice point for $\alpha$. I am not postulating that there are choice points or non-vacuous choices, but of course unless this is so there is no point to this theory except to explain what the structure of agency would be if there were any.

There are two further postulates.

(T10) When considering multiple agents, I postulate for each moment that for each way of selecting one possible choice for each agent from among his or her set of choices, the intersection of all the possible choices selected must contain at least one history (Something happens).

Sometimes this is thought of as "independence of choices", which is a good thought. At any one moment, $w$, the choices possible to each agent are indeed independent in the sense, for example, that any possible choice, choice $\alpha_{\alpha}^{w}\left(h_{1}\right)$, that $\alpha$ makes is consistent with any possible choice, choice ${ }_{\beta}^{w}\left(h_{2}\right)$, that $\beta$ makes: the intersection of these two sets must be nonempty. Since the choices are simultaneous it is certainly reasonable to think of them as independent. What might be confusing about the language of independence is this: the entire set of choices, choice $_{\alpha}^{w}$, open to $\alpha$ at $w$ is by no means "independent" of the entire set of choices, choice ${ }_{\beta}^{w}$, open to $\beta$ at $w$. The very fact that each member of the first set must have nonempty overlap with each member of the second is itself a fierce constraint. It is easy to see, for instance, that no two agents can possibly have exactly the same possible choices at exactly the same moment (vacuous choice aside).

The final postulate requires the previously given temporal-modal definition of "undivided".

(T11) If two histories are undivided at $w$, then no possible choice for any agent at $w$ distinguishes between the two histories. One of two histories undivided at $w$ belongs to a certain choice possible for $\alpha$ at $w$ if and only if the other belongs to 
exactly the same possible choice (No choice between undivided histories). ${ }^{14}$

It follows that if two histories are choice-distinguished for any agent at $w$, then they must split at $w$. It also follows that if two histories have no moment of splitting, then they must be choice-equivalent for every agent at every moment through which they both pass. If two histories have no moment of splitting, then no agent can ever make a choice that distinguishes between them. Only non-agentive processes can tell them apart. ${ }^{15}$

Another important consequence is that from the point of view of a properly later moment, $m$, what choice an agent made at each properly earlier moment, $w$, is uniquely determined. Or to say the same thing from the point of view of $w$, for each moment, $m$, properly later than $w$, there is a unique possible choice for $\alpha$ at $w$ that contains all histories passing through $m$. For argument, assume $m$ is properly later than $w$. Then the two moments constitute a (two-member) chain that by the Axiom of choice can be extended to a maximal chain, i.e., a history, $h$, that contains them both. Existence is partly given by choice ${ }_{\alpha}^{w}(h)$, which evidently contains a history, namely, $h$ itself, that passes through both $w$ and $m$. To continue with existence, let $h^{\prime}$ be any history through $m$, and hence, by No backward branching, through $w$. By No choice between undivided histories, $h^{\prime}$ must belong to choice ${ }_{\alpha}^{w}(h)$, which then contains every history through $m$. As for uniqueness, if any possible choice for $\alpha$ at $w$ contained all histories through $m$, it would also contain $h$, which would imply its identity with choice ${ }_{\alpha}^{w}(h)$, as required.

Provided $m$ is properly later than $w$, this fact justifies introducing the defined notation, $\operatorname{choice}_{\alpha}^{w}(m)$, for the possible choice for $\alpha$ at $w$ that contains all histories running through $m$. This set of histories is "the choice that was made by $\alpha$ at $w$ ", where the past tense is issued from the point of view of $m$. The point of the notation is clearly to exhibit that the choice that was made by $\alpha$ at $w$ (with past tense from the point of view of $m$ ) is quite independent of which history through $m$ is considered; for instance, choice $_{\alpha}^{w}(m)$ is equally definable as the possible choice for $\alpha$ at $w$ that contains some history through $m$. We should also remark the following confusing feature of the notation: choice $_{\alpha}^{w}(m)$ is always a subset of $H_{(w)}$, but not in general a subset of $H_{(m)}$.

That is the end of those postulates concerning the metaphysics of agency, temporality, and modality that I think are required for a pre- 
liminary understanding of stit. All else is more concepts, some metaphysical, some semantic.

\section{WITNESS OF STIT BY MOMENTS}

It is time to begin discussing the semantics of agency. I wholly follow Thomason in believing that a semantics in the context of historical indeterminism needs to make truth (and also denotation!) relative to moment/history pairs. This is the fundamental, inescapable, often unmemorable point. Sometimes it is convenient to take the shortcut proposed by Thomason 1970 and say that a sentence is true directly relative to a moment, $m$, (without mention of a history) if it is true at every pair $m / h$, where $h$ is one of the histories passing through $m$, but sometimes it is more unsettling than convenient; in the present discussion I partially use the shortcut by saying that a sentence is settled true at $m$ (without mention of a history) if it is true at every pair $m / h$ with $h$ passing through $m$. In addition I will say that such a sentence is also settled true at each of the moment/history pairs involved. Ditto for settled false. On my usage, then, one may drop reference to a history in the context of a phrase "settled true" or "settled false", but my usage does not permit dropping reference to a history for plain "true" or "false". ${ }^{16}$

Sometimes my plan, or that of Thomason, is described as a willingness to countenance sentences or perhaps propositions without a truth value. This is wrong: every sentence has a truth value with respect to every moment/history pair. What is right is that the moment alone does not determine a truth value (and neither does the history alone). What throws us off balance is that the context of utterance gives us only the moment, since (unless determinism be permanently true) the moment of utterance is part of many histories. Lewis 1986 thinks otherwise, or at least I think he does: " $\mathrm{it}^{17}$ alone is influenced causally by what we do and how we are in the present". The disagreement is, I take it, over whether the phrases employed in fact pick out a unique history. With respect to the present account of the "influence" of "what we do", uniqueness is contrary to its very presuppositions, and in particular, there is no "what we do" if there is a unique future history through our present moment.

I am going to extend the temporal-modal analysis before offering a "final" semantic account of agency, but it will be helpful if first I 
rehearse the semantics of Belnap and Perloff 1989 so that I can point to the specific shortcoming that motivates the extended analysis.

[ $\alpha_{\text {stit: }} Q$ ] is true at $m / h$ (where $h$ passes through $m$ ) just in case there is a moment, $w$, that is a momentary witness to $[\alpha$ stit: $Q]$ at $m$, where that phrase is defined by three conditions.

- Priority. $w$ is properly earlier than $m$.

- Positive condition. $Q$ is settled true at every moment in $i_{(m)}$ that lies on a history in choice $_{\alpha}^{w}(m)$.

A moment in $i_{(m)}$ that lies on a history in $\operatorname{choice}_{\alpha}^{w}(m)$ may be said to be choice-equivalent to $m$ for $\alpha$ at $w$. Thus the Positive condition is that $Q$ should be settled true at every moment choice-equivalent to $m$ for $\alpha$ at $w$. In other words, the choice that $\alpha$ made at $w$ (where the past tense is from the perspective of $m$ ) "guaranteed" that $Q$ would be settled true at every moment of $i_{(m)}$ accessible from $w$ via that choice (where the subjunctive is also from the point of view of $m$, since I am considering alternate ways of filling the same instant).

- Negative condition. $w$ must lie under some moment $m^{\prime}$ in $i_{(m)}$ at which $Q$ is not settled true.

Thus, the choice that $\alpha$ made at $w$ was not irrelevant to the truth of $Q$ at $i_{(m)}$ in the sense that at $w$ the falsity of $Q$ at $i_{(m)}$ is risked - its settled truth there is not guaranteed.

Since neither the positive nor negative conditions mention $h$, it is clear that $[\alpha$ stit: $Q]$ is either settled true or settled false at each $m .{ }^{18}$

\section{WITNESS OF STIT BY CHAINS}

A deficiency in the concept of stit just defined is that it makes it a matter of "logic" that if Autumn Jane saw to it that she was clean at 4:00, then there was some momentary choice that witnessed that fact. And maybe there was; but suppose instead that the witness to the outcome was a chain of choices by Autumn Jane with no last member. Picture her just prior to 4:00 as balancing on a board that crosses over a puddle, and award her the ability to choose to fall off at any of an unending series of moments approaching 4:00. Also permit my illustration to be simple by adding what is probably false: that the lapse 
from cleanliness occurs as soon as you like after the choice to fall off. Under this supposition the witness to her seeing to her own cleanliness at 4:00 was no single choice, but the whole unending chain of choices properly approaching $4: 00 .{ }^{19}$

To keep us all on the right track, I explicitly note that the deficiency to which I point is not all carried by stories in which in ordinary speech we would say that a long list of preliminary activities went into, say, Autumn Jane's setting of the table, or to change the illustration in order to make the point even more visible, a story in which in order for Autumn Jane to win at chess at 5:00, a complicated, temporally discrete series of moves was required. The chess illustration makes it clear that catering to these stories has nothing to do with an unending sequence of choices. Reflection on the conceptual problems raised by such stories is important, but these problems are so different from those that I am now discussing that it will require a different occasion; for now I only mention the relevance of the delicate notions of strategy and of seeing to it that one can later see to it.

I return to the quest for an understanding of the witness of a stit by a possibly unending sequence of choices. In Belnap 1989, note 13, I took a short path to the goal; here I take a longer but I think more enlightening route.

We work for a while with chains (sets all of whose members are comparable). For orientation, we define the following kinds of chains (in addition to histories, future histories, and pasts). A semi-interval is a (possibly empty) upper-bounded chain that contains every moment that lies between any two moments it contains; let $n$ range over semiintervals. An interval is a lower-bounded semi-interval; so every semiinterval is either an interval or a past. Semi-intervals may be upper closed (i.e., have and contain a least upper bound) or not; intervals may in addition be lower closed (with a dual definition) or not.

Given a chain, $c$, there are two sets of histories related to it in which we might be interested. To avoid any subsequent hesitation, I will use $H_{(c)}^{\exists}$ for the one in which we will be most interested, the set of those histories passing through some member of $c$. We can use $H_{(c)}^{\forall}$ for the set of histories that traverse $c$, i.e., that pass through all moments in $c$.

In a critical definition, given a chain, $c$, I say that two histories are choice-equivalent for $\alpha$ at $c$ just in case first, they both belong to $H_{(c)}^{\exists}$ (so surely some member of $c$ belongs to both histories), and second, 


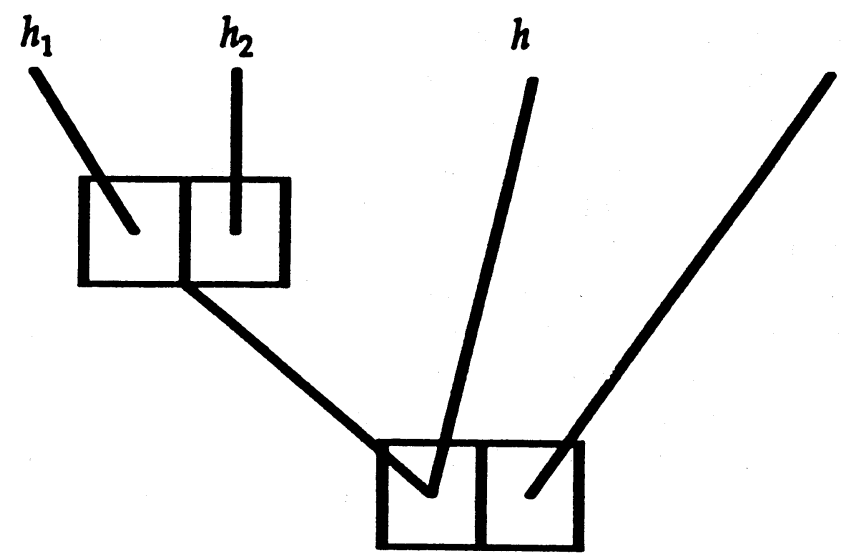

Fig. 2. Non-transitivity of choice-equivalence at a chain.

no member of $c$ choice-distinguishes the two histories for $\alpha$ (no choice that $\alpha$ makes in the course of $c$ distinguishes the two histories). Evidently the only way two histories in $H_{(c)}^{\exists}$ can fail to be choice-equivalent for $\alpha$ at $c$ is if $c$ contains the very moment of splitting of those two histories, and if at that moment they belong to different possible choices for $\alpha$. Warning: in spite of what I think is well-chosen language, choiceequivalence is not an equivalence relation on $H_{(c)}^{\exists}$ : it is not transitive. The following is an example, where the chain, $c$, consists of the two moments represented by divided rectangles. The division of each rectangle represents that at each moment there are two possible choices for $\alpha$.

In this diagram the history, $h$, that splits from the chain at the lower moment is choice-equivalent for $\alpha$ to $c$ to each of the histories $h_{1}$ and $h_{2}$ that split at the top moment; but obviously $h_{1}$ and $h_{2}$ are not choiceequivalent for $\alpha$ to $c$ to each other. That is, no choice that $\alpha$ makes in the course of $c$ distinguishes $h$ from $h_{1}$, or $h$ from $h_{2}$, but there is evidently a choice (at the top moment) that distinguishes $h_{1}$ from $h_{2}$. So choice-equivalence for $\alpha$ at a chain is not and should not be transitive.

For each $h$ in $H_{(c)}^{\exists}$, let choice ${ }_{\alpha}^{c}(h)$ be the set of histories choiceequivalent to $h$ for $\alpha$ at $c$. Further, suppose that $m$ is properly later than every moment in $c$. Then in fair analogy to what we have done before, we may define choice ${ }_{\alpha}^{c}(m)$ as the set of histories in $H_{(c)}^{\exists}$ that are choice-equivalent to every history through $m$. 
Chains are "vertical". To call attention to a key "horizontal" set of moments, I define $M_{(w, i)}$, the horizon from moment $w$ at instant $i$, as the set of moments at which histories through $w$ intersect $i$. In all the cases that $\mathrm{I}$ have in mind, $w$ precedes $i$, so that the picture is that the horizon is the "base" at $i$ of an inverted (downside up) isosceles triangle whose apex is underneath at $w$. I then say that it is determined at $w$ that $Q$ is settled true at $i$ iff $Q$ is settled true throughout the horizon $M_{(w, i)}$ from moment $w$ at $i{ }^{20}$ As one looks into the future from the perspective of $w$, there is no escaping that $Q$ is settled true at every spot on the horizon provided by $i$. To idealize: having posted the letter, it is just now determined that "the letter is delivered" is settled true tomorrow, regardless of whether it rains or shines, of whether there is a sea-battle or not, etc.

Take a moment $m$ and a sentence $Q$. Assume that $Q$ is settled true at $m$. Either $Q$ has been determined to be settled true at $i_{(m)}$ from all eternity (a vertical picture), which is just the same as saying that $Q$ is settled true at each and every moment in $i_{(m)}$ (this, by Historical connection, is an equivalent horizontal picture), or else the opposite. If the opposite, let $p$ be the set of moments $w$ prior to $m$ such that it is false that it is determined at $w$ that $Q$ is settled true at $i_{(m)}$. Evidently $p$ is a past; I call it the upper cusp for $Q$ at $m$, since on the one hand the fact that $Q$ is settled true at $i_{(m)}$ is not determined at any moment in $p$, while on the other hand, at every moment after $p$ up to and including $m$ itself, it is determined that $Q$ is settled true at $i_{(m)}$. Thus, where $p$ draws to a close is the very "point" at which the status of $Q$ changes from "not yet determined to be settled true at $i_{(m)}$ " to "now determined to be settled true at $i_{(m)},{ }^{21}$ I observe that the upper cusp is located in total independence of how the sentence, $Q$, itself may change its truth value as it passes along a history from one moment to another.

The upper cusp for $Q$ at $m$ is defined independently of agency; it might therefore be called the cusp of causality, thinking now of causality as a relation between a settled fact such as that represented by $Q$ at $m$ and an "event" in the sense of a chain of moments (instantaneous slices). The idea is that nothing can be an effect except what changes from not being determined to be settled true to being determined to be settled true, and that the locus of causality must be at the "point" at which this change takes place: the upper cusp, or cusp of causality is just where the effect comes to be determined to be settled true. You will perhaps be made suspicious by noticing that the suggestion that an 
interesting cause/effect relation is between an "event" as cause and a "fact" as effect is driven by theoretical metaphysics rather than by linguistic or common sense analysis; but surely it won't hurt to think about it, not even if you notice that if determinism be true, then causality in this sense does not exist!

There is or may be another critical "point", this one tied to agency. Given $Q$ settled true at $m$, I say a subpast $p$ of $p_{(m)}$ precedes any possible witnessing of $[\alpha$ stit: $Q]$ at $m$ if there is some moment $m^{\prime}$ in $i_{(m)}$ at which $Q$ is not settled true such that (a) $p$ is the set of all common lower bounds of $m$ and $m^{\prime}$ and (b) $m$ and $m^{\prime}$ are choice-equivalent for $\alpha$ at every single member of $p .^{22}$ During such a past is not when $\alpha$ can make choices that guarantee that $Q$ is settled true at $m$, for any such choice would permanently entangle $m$, where $Q$ is settled true, with some other co-instantaneous moment, $m^{\prime}$, at which $Q$ is not settled true, as far as $\alpha$ is concerned. The adverb "permanently" is appropriate because $p$ was chosen as the set of all common lower bounds of $m$ and $m^{\prime}$, so that we can be sure that no later moment can break the choiceequivalence. The union of all subpasts of $p_{(m)}$ that precede any possible witnessing of [ $\alpha$ stit: $Q$ ] at $m$ is itself a past, unless it is empty; this union is the lower cusp for $[\alpha$ stit: $Q]$ at $m$.

Here is another route to the same concept. Say that two co-instantaneous moments are choice-equivalent for $\alpha$ if they are choice-equivalent for $\alpha$ at every point in their common past. Nothing that $\alpha$ can ever choose distinguishes between two such moments. Say next that a moment, $m^{\prime}$, that is co-instantaneous with $m$ is a threat to [ $\alpha$ stit: $\left.Q\right]$ at $m$ that cannot be removed by a choice of $\alpha$ if (i) $m^{\prime}$ is choice-equivalent to $m$ for $\alpha$ and (ii) $Q$ is not settled true at $m^{\prime}$. The thought is that since nothing $\alpha$ chooses can remove such a threat, no choice that $\alpha$ makes while such a threat still lies ahead can count toward the truth of $[\alpha$ stit: $Q$ ] at $m$. The removal of such threats can only come (if they do) by processes out of the control of $\alpha$; only after they are all removed can $\alpha$ begin to make choices testifying to the truth of $[\alpha$ stit: $Q]$ at $m$. Thus, the lower cusp for $[\alpha$ stit: $Q]$ at $m$ is the set of all moments that lower bound both $m$ and some moment, $m^{\prime}$, that is a threat to $[\alpha$ stit: $Q]$ at $m$ that cannot be removed by $\alpha$.

Keeping $p$ as the upper cusp for $Q$ at $m$, let $p^{\prime}$ be the lower cusp for [ $\alpha$ stit: $Q]$ at $m$. Here is the central doctrine: the only room for $\alpha$ to make choices guaranteeing the settled truth of $[\alpha$ stit: $Q]$ at $m$ is the chain of moments that are both members of the upper cusp and properly 
upper bound the lower cusp; that is, the uniquely determined locus of choice for $[\alpha$ stit: $Q]$ at $m$ is just $p-p^{\prime}$. Notation: $n([\alpha$ stit: $Q], m)$, which is a semi-interval, is the locus of choice for $[\alpha$ stit: $Q]$ at $m$. This semiinterval may be empty. If and only if it is not empty (including the case when it contains a single point), I call it a witness to $[\alpha$ stit: $Q]$ at $m$.

The word "permanently" in the last-but-two paragraph is not only appropriate but significant. There is nothing that prevents a witness, as just defined, from containing some moments that are perhaps even vacuous choices for $\alpha$, as long as the witness also contains later moments that do more work. Thomson 1977, pp. 51-57, tells a pair of entirely apt stories, one of herself cleaning a kitchen floor with Stuff, the second of her neighbor using Super-stuff. The difference is this: Stuff nearly completes the cleaning all by itself but leaves a bit of dusty residue that needs to be removed, whereas Super-stuff wholly completes the job without intervention. In each case the person might as well sleep while the chemical does its cleaning - on the present system of bookkeeping, no credit would be lost towards seeing to it that the floor is clean. Thomson further argues that there there is no difference between the two stories if what one is after is what events are in each case part of the agent's cleaning of her floor; it is clarifying to observe that the current apparatus, at least as so far developed, is not capable of answering this question. Nevertheless, for better or worse, the stories yield different loci of choice for the floor's being cleaned by the respective agents: in the Stuff case the fact that there are choices yet to be made (when the residue is being swept up) permits the inclusion in the locus of choice of the moments during which Stuff is working, whereas in the Super-Stuff case, when Super-Stuff is fully spread on the floor, the moments of choice are fully past: it is then determined that it is settled that the floor comes clean. These moments when Super-stuff is inexorably doing its super thing are therefore not permitted to be part of the locus of choice, whether or not they are to be counted as part of an event to be identified as the agent's seeing to it that the floor is clean.

The previous definition said only when the locus of choice was a witness. We approach the same matter from a different direction by giving an account of a witness by any chain, $c$, to $[\alpha$ stit: $Q]$ at $m$. Four conditions must be satisfied by $c$.

- $\quad$ Priority. All moments in $c$ must be properly earlier than $m$.

- Nonemptiness. The chain, $c$, must of course be nonempty. 
- $\quad$ Positive condition. $Q$ must be settled true at every moment in $i_{(m)}$ that lies on a history in choice ${ }_{\alpha}^{c}(m)$.

- Negative condition. Every moment, $w$, in $c$ "risks" the falsity of $Q$ in the sense that there is above $w$ some moment in $i_{(m)}$ at which $Q$ is not settled true. In other words, for every moment, $w$, in $c$, it is not determined at $w$ that $Q$ is settled true at $i_{(m)}$.

We may then identify $n_{([\alpha \text { stit: } Q], m)}$, provided it is a witness, as a maximal witness to $[\alpha$ stit: $Q]$ at $m$, which is to say that (1) it satisfies the Positive and Negative conditions, and (2) has no proper superchain that satisfies these conditions (Maximality condition).

It is clear that the Maximality condition is of secondary interest; it is what makes the locus of choice unique, but that uniqueness itself is (as far as I know) of largely technical interest. The fact is that it is always the upper cusp at which there goes on the work of changing $Q$ from not-settled true to be settled true. Any nonempty upper stretch of the locus of choice for [ $\alpha$ stit: $Q$ ] at $m$ is doubtless equally entitled to bear the name of witness, as indeed is any nonempty chain whatsoever that satisfies the Positive and Negative conditions. Nevertheless it remains true that if any nonempty subset satisfies the Positive and Negative conditions, then the locus of choice is itself a witness to [ $\alpha$ stit: $Q$ ] at $m$. It follows that although whether or not to admit these other subsets may make a difference to those philosophers whose task is to have an ontology of actions as a species of event (whose task, for instance, includes finding an event to be identified with the agent's seeing to it that the floor is clean), it does not make a difference for the modal theory of agency. ${ }^{23}$

It nevertheless seems a good idea to go into the matter a bit more deeply. One approach that seems helpful is this. Suppose that a chain, $c$, is a witness to [ $\alpha$ stit: $Q$ ] at $m$. What can we add to or subtract from $c$ and still have a witness?

There are three things to say about addition, for we can think about adding moments that are (1) later than, (2) in the middle of, or (3) earlier than the chain, $c$.

(1) No moment, $w$, that is properly later than $c$ can be added, essentially because of No choice between undivided histories: any history that testified to the satisfaction of the Nega- 
tive condition by $w$ would also testify to the failure of the Positive condition for $c$.

(2) Any intermediate moment, $w$, can be added to $c$. The addition could not cause a failure of the Negative condition, because satisfaction of that condition obviously propagates downward. And the addition cannot generate a failure of the Positive condition because (a) the set of histories needing to be checked for choice-equivalence remains the same (i.e., $H_{(c)}^{\exists}=H_{(c \cup\{w\})}^{\exists}$, and (b) adding a moment cannot make a relevant history choice-equivalent if it was not so before.

(3) Earlier moments can sometimes be added and sometimes not, and sometimes need to be added in batches (entire pasts) if at all. Certainly no moment in the lower cusp can be added; as noted previously, any such attempted addition would cause a failure of the Positive condition. Suppose we wish to add a moment $w$. We have to worry about all moments, $m^{\prime}$, in the horizon from $w$ at $i_{(m)}$ that are choiceequivalent to $m$ for $\alpha$ at $w$ and at which $Q$ is not settled true. We can add $w$ provided (1) we also add for each of these moments, $m^{\prime}$, its least upper bound $w^{\prime}$ with $m$ (all bets are off - we cannot add $w$ - if it doesn't have one), and provided (2) $w^{\prime}$ choice-distinguishes $m$ from $m^{\prime}$ for $\alpha$, and provided (3) we keep (successfully) carrying out the same process for the newly added moments $w^{\prime}$.

There are two fundamental things to be said about preservation of witnesshood under subtraction.

(1) Say a moment $w$ is a choice point for $[\alpha$ stit: $Q]$ at $m$ if $w$ is the greatest lower bound of $m$ and some other moment $m^{\prime}$ in $i_{(m)}$ at which $Q$ is not settled true, provided that $m$ and $m^{\prime}$ are not choice equivalent for $\alpha$ at $w$. (This last must be true by the Positive condition if the choice point is a member of a witnessing chain.) Also call a moment vacuous for $[\alpha$ stit: $Q]$ at $m$ if it is not a choice point. Then if $c$ is a witness for $[\alpha$ stit: $Q]$ at $m$, so is the result of removing all the vacuous moments from $c$, leaving just the choice points. This is a considerable step in the direction of a minimal witness when that is what is found interesting, and if we carry out this subtraction on $n([\alpha$ stit: $Q], m)$, we obtain another candidate for "the" locus of choice. 
The other side of this first observation is that one cannot subtract a choice point unless one also is prepared to subtract all its predecessors, for otherwise the No choice between undivided histories condition would imply that some earlier moment failed the Positive condition.

(2) Any proper initial (i.e., early stretch) of a witness can be subtracted at will. When the witness has no last member, this evidently does nothing whatsoever for uniqueness. When, however, the witnessing chain has a last member, one can subtract every member of the witness except that last member, leaving a unit set. Nothing could be more minimal than that, and in this special circumstance, we have a third candidate for "the" locus of choice. In such a case we may as well speak of witness by the moment itself: momentary witness, as we will say.

\section{SEMANTICS FOR STIT}

At this point the chain semantics for stit are anti-climatic, all the work having gone into the underlying metaphysics. The following are equivalent definitions.

- $\quad[\alpha$ stit: $Q]$ is true at a moment/history pair $m / h$ just in case the locus of choice, $n_{[[\alpha \text { stit:Q],m) }}$, is nonempty and is therefore a witness to $[\alpha$ siti: $Q]$ at $m$.

- $\quad[\alpha$ stit: $Q]$ is true at a moment/history pair $m / h$ just in case there is a chain, $c$, satisfying the Priority, Nonemptiness, Positive, and Negative conditions for $c$ to witness $[\alpha$ stit: $Q]$ at $m$.

I will have a go at restating a variation on the second definition in just slightly different language. For $[\alpha$ sit: $Q]$ to be true at $m / h$ there must be a nonempty prior chain of choice points for $\alpha$ satisfying two conditions. (i) At each member of the chain, $Q$ at $i_{(m)}$ is at risk (it is not yet determined that $Q$ should be settled true at $i_{(m)}$ ). (ii) Each of those places in $i_{(m)}$ at which $Q$ is not settled true that lie above some member of the chain is eventually weeded out by an actual choice that $\alpha$ makes in the course of the chain (it is not eliminated just by the choice of other agents, or by non-agentive processes, or by the mere passage of time). The idea is that if no choice that $\alpha$ makes in a chain distinguishes between two moments, then one could not truly say that the chain 
witnesses [ $\alpha$ stit: $Q]$ at one but not the other. Hence, if $Q$ is not settled true at one, the chain cannot witness $[\alpha$ stit: $Q]$ at the other, for $[\alpha$ stit: $Q]$ should imply $Q$.

It would be appropriate next to elaborate on the provable properties of stit, with a special eye on the Refref conjecture. As announced at the outset, however, another occasion will be required in order to go into the matter of whether Autumn Jane can refrain from refraining from seeing to it that she keeps clean other than by seeing to it that she keeps clean. Instead I make two brief statements as to what I know about the Refref conjecture, and then close with some brief remarks on a topic on which Prior has spoken with his unfailing insight.

The first statement is that the denial of the Refref conjecture is consistent with the postulates we have so far set down. For a picture see Figure 10 of Belnap 1989.

The second statement is that with an additional hypothesis, the Refref conjecture is true. The hypothesis is this: there are no Busy Choosers, where an agent is defined as a Busy Chooser if he or she makes infinitely many nonvacuous choices between two given moments. If there are no Busy Choosers, then the Refref thesis is true; and contrapositively, if a story makes the Refref thesis false, then that story involves a Busy Chooser. For a picture relevant to a proof, see Figure 9 of Belnap 1989.

\section{CONTEMPLATION AND ACTION}

I close, as promised, with brief consideration of a thesis of Prior. The thesis of Chapter IV of Prior 1968 is that contemplation and action are incompatible in the sense that no one can find out what anyone is going to decide - under the assumption that there really is action and not just its appearance. I think Prior's argument is absolutely good; all I do is lay out what I take to be its essential elements in present terminology. The most interesting thing thereby revealed is how little philosophy of mind or epistemology is needed.

First the concept of "finding out". I substitute a verb that is philosophically somewhat more familiar, the verb "to know". To make the argument as simple as possible, let us fix the knower as Autumn Jane, and let us consider the claim that "Autumn Jane knows that her father will see to it that her dress is clean". I introduce just two postulates 
governing the semantics of knowledge, both of which I think are implicit in the Prior argument.

The first postulate about knowledge is one whose point can only be appreciated by keeping in mind the branching of the future: when we have knowledge, it is settled true that we have it. If at a given moment/history, $m / h$, Autumn Jane knows that $Q$, then this does not depend on the history, $h$, but must be equally true for every history passing through $m$. This postulate says that knowledge is what we may honor Carnap by calling moment-determinate: regardless of $Q$, for each moment, $m$, "Autumn Jane knows that $Q$ " is true on any pair $m / h$ just in case "Autumn Jane knows that $Q$ " is true on every pair $m / h$ ', with the moments the same and with the histories passing through the moment.

In this way knowledge is quite an ordinary property, like "red" or "six feet tall", and it would appear that the moment-determinateness of knowledge hardly needs a special argument; it has nothing to do with the distinctive character of knowledge as such. In fact, just about any standard epistemic, doxastic, or psychological attitude has exactly the same character of moment-determinateness. If Autumn Jane believes (wonders, has asserted, predicted or guessed) something, then that she has that belief (is in that state of wonder, has made that assertion, prediction or guess) does not depend on what the future may bring; it is a settled truth about her. In particular, the momentdeterminateness of knowledge has nothing to do with the characteristic property of knowledge that it is supposed to be "justified".

Prior on p. 36 rightly indicates that it makes a difference that knowledge is different from correct guessing or (to change the wording) true belief. The peculiarity, curiously enough, is on the side of the compound: although knowledge and belief are alike in being momentdeterminate, true belief is not moment-determinate - just because truth is not! Nor does adding justification help: the state of being justified in a belief is plausibly moment-determinate, but to add "and it is true", as required by the phrase "justified true belief" is to break the momentdeterminateness. Thus, if I am right, the puzzle about knowing what you are going to do does not arise for having a true, justified belief about what you are going to do; the compound is not moment-determinate.

The second postulate is that knowledge implies truth, by which I mean this: for every moment/history pair, if "Autumn Jane knows that 
$Q$ " is true at that pair, so is $Q$. Given that she knows that her father will see to it that her dress is clean, then in fact her father will see to it that her dress is clean. I do not think that the problem with which Prior is wrestling is taken by anyone to be a problem unless we have this one among the philosophers' senses of "knowledge" in mind; if knowledge only conveys justified belief or some justified level of certainty or some justified certainty as to high probability, or if it merely tends to signal truth but does not betoken an exception-free universality, then there is no puzzle in supposing that Autumn Jane "knows" what someone is going to do. The puzzle only sets in when it is supposed that knowledge guarantees (implies) truth.

It immediately follows from these two postulates that if at $w / h$ Autumn Jane knows that $Q$, then $Q$ is settled true at $w$. By contraposition, if $Q$ is not settled true at $w$, then on no history through $w$ does Autumn Jane know that $Q$. It is therefore impossible that Autumn Jane should know something that is not settled true. She can consistently be said to believe it, wonder if it is so, assert it, guess it, etc., for although each of these shares moment-determinateness with knowledge, none of these have the second property, that of implying truth. But since knowledge does have this second property, she cannot consistently be said to know a truth that is not settled true. Thus, if Autumn Jane knows that her father will see to it that her dress is clean, then it is settled true that her father will see to it that her dress is clean.

Given this intermediate conclusion, it is obvious what we need about the concept of decision: nothing about the torments of the mind in process, but only the colorless fact that at or before the point of decision it is not settled what decision is made. On the present theory the required true premiss could be stated as follows: if a moment, $w$, is in or precedes the upper cusp for $Q$ at $m$, then at $w$ it is not settled that it will be the case that $Q$ at $i_{(m)}$, and therefore not settled that it will be the case that $[\alpha$ stit: $Q]$ at that instant. This is the most elementary of consequences of the Negative condition.

With these three nearly mindless premisses, the conclusion that what someone does cannot be known until the upper cusp is past is nothing but a modus tollens: when "Autumn Jane's father will see to it that her dress is clean" is known, then it is settled true; but "Autumn Jane's father will see to it that her dress is clean" is not knowable by Autumn Jane - or anyone else - until after the upper cusp.

Just to double underline the point we are urging, which is that the 
argument does not depend on interesting commitments about the Mind, we repeat the premisses and the conclusion. (1) Like all standard propositional attitudes, knowledge is moment-determinate. (2) Knowledge (in the sense that gives rise to Prior's puzzle) implies truth. (3) No stit proposition is settled true until after its upper cusp. Therefore, (4) it is impossible to have knowledge of a stit proposition before its upper cusp is past.

\section{NOTATION}

More henscratches have been introduced than are good for any one paper; here, for reference, is a list.

\section{〈Tree, $\leqslant$, Instant, Agent, choice〉}

\section{fundamental structure}

$<$

properly earlier than

$m, w$ moments (members of Tree)

$c$ chain (of moments)

$h$ history (maximal chain of moments),

$H$ set of histories

$H_{(m)}$

$H_{(c)}^{\exists}$

$H_{(c)}^{\forall}$

set of histories through $m$ set of histories passing through some member of $c$ set of histories passing through every member of $c$ past (maximal upper bounded chain of moments)

$p^{*}$

$p_{(m)}$ the completion of $p$ (add any least upper bound) set of all moments up to and including $m$ instant (member of Instant) the instant to which $m$ belongs the moment in which $i$ intersects $h$ set of moments the horizon from $w$ at $i$ (the set of moments in which histories through $w$ intersect $i$ )

$n$

$\alpha$ $\operatorname{choice}_{\alpha}^{w}$ $\operatorname{choice}_{\alpha}^{w}(h)$ $\operatorname{choice}_{\alpha}^{w}(m)$ semi-interval (interval or past) agent (member of Agent) the set of possible choices for $\alpha$ at $w$ (a partition of $\left.H_{(w)}\right)$

the possible choice for $\alpha$ at $w$ to which $h$ belongs (assuming $h$ is in $H_{(w)}$ )

the possible choice for $\alpha$ at $w$ to which every history through $m$ belongs (assuming $w<m$ ) 


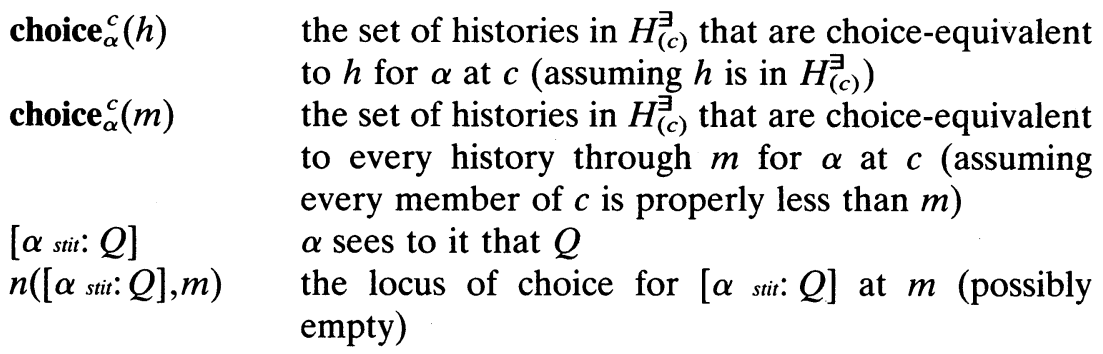

\section{NOTES}

1 Thanks to W. Lycan for correspondence prompting some of the discussions herein, to M. Xu and P. Bartha for catching several errors and to M. Perloff for repeated help.

${ }^{2}$ The approach to agency via a modality is in contrast to the familiar effort to understand agency by means of the reification of actions as a species of events. For a mini-history of the topic, see Belnap 1989.

${ }^{3}$ For those who do not care much about the Refref conjecture, various other ways in which the stit analysis helps us to understand agency are illustrated in Belnap 1988, Belnap and Perloff 1989, and Belnap 1989.

${ }^{4}$ It is not likely that the present theory will be able to take account of general relativity without substantial alterations, and perhaps the same is true of quantum theory; but these are not areas in which I am at home. All I can add is a conviction that it will not be possible to make suitable advances without consideration of the work of Bressan 1972, 1974 , 1989, and Zampieri 1982, 1982-83, for they are the only persons I know who have worked within what seems to me the only reasonable position, that identifying eventpoints across possible situations is neither trivially easy (perhaps Kripke thinks this) nor a matter of partial constraint and partial stipulation (perhaps Lewis thinks this) nor empirially insignificant (perhaps, to keep to one department, this is van Fraassen's view), but a matter of serious physics.

5 The tree concept in modern tense logic is due to Prior 1967. I learned it mostly from Thomason 1970 (the best account is in Thomason 1984) and McCall 1976 (current important work by McCall on the topic is in process, some of which I have been privileged to see). I note that Thomason, with his eye on subjunctive conditionals and epistemic possibilities, makes no special place for Tree as here limited by "historical connection" (defined below), and that McCall quite rightly worries about the reality of what might have been. As for the appreciation of the relevance of the tree concept to agency, there is no overestimating the influence of von Neumann and Morgenstern 1944. For another valuable line of research into agency based on the arboreal insights of Prior and von Neumann, consult Aqvist 1974, 1978 and related papers, including Mulloch 1988. I also am happy to acknowledge a particular indebtedness to the deeply philosophical efforts of Hamblin 1987 to formulate an "action-state semantics" in terms of a tree-like structure. ${ }^{6}$ See especially McCall 1976.

${ }^{7}$ If one makes a cut of a history into two disjoint and exhaustive parts, $\langle p, f\rangle$, such that each element of the first is less than each element of the second, then $p$ is a past (history) and $f$ is a future history. If in a partial analogy one makes a cut of the entire Tree into 
two disjoint and exhaustive parts, $\langle p, f\rangle$, such that each element of the first is less than each element of the second, and if one imposes the additional requirement that $p$ be a past (history), then $f$ is a future.

${ }^{8}$ This simply adapts to singular terms the sentential semantic insight of Thomason 1970.

${ }^{9}$ Thomason in the passage cited is describing, not endorsing.

${ }^{10}$ For reflecting on such things, it is good to keep in mind that if $h$ and $h^{\prime}$ are distinct histories, then $\left\langle h \cap h^{\prime}, h-h^{\prime}\right\rangle$ is a cut of $h$ : the left and right sets are each nonempty, they have an empty intersection, they sum to $h$, and each moment in the left set is properly less than each moment in the right set.

${ }^{11}$ Just to make things clear (but not to argue) by an example, I am disallowing that it is or was really possible that there should be blue swans unless there is some moment in our past that has a moment future with respect to it at which there are blue swans. I therefore doubt that it is easy to be sure that it is or was really possible that there should be blue swans. Of course something terminological is going on here: I am using "really possible" as what is or was determined as possible in the world of our context of utterance, and thus in a sense much narrower than that sought by e.g. Lewis 1986 through the idea of recombination. But there is also something non-terminological: I think that the Humean picture of enormous recombinational possibilities for the immediate future (e.g. blue swans on my desk one nanosecond from now - Lewis 1986, p. 91, says that "anything can follow anything") is not relevant to what can be seen to, and that instead what counts is only the current - much narrower - set of real possibilities. But I repeat: I am attempting to clarify rather than argue.

${ }^{12}$ Evidently my uses of "moment" (in which I follow Thomason) and "instant" are jargon not sanctioned in ordinary speech, although the distinction is certainly there to be drawn.

${ }^{13}$ There is a more general theory, essentially suggested by Gupta: take the second argument of choice to be a complete past instead of a moment. This suggestion has not been fully explored; it would seem to suggest a matching generalization of semantic relativization, and a consequent need to study the problem of adding Instant* to the completing Tree* of Tree, but I, for one, am hazy on this topic.

${ }^{14}$ I learned the No choice between undivided histories condition from P. Kremer in 1987. All the postulates having to do with choices are to be found in one form or another in von Kutschera 1986.

${ }^{15}$ This is the consequence of the present theory that the Gupta generalization suggested in note 13 was designed to avoid.

${ }^{16}$ My usage here is in structural agreement with that I suggest as best for formulas with free variables. Thus, I will say that "Fx" is universally true or universally false (or neither), without mention of an assignment of values to the variables, but I do not permit myself to say that " $\mathrm{Fx}$ " is just plain true or plain false. I don't mean that one could not or must not say such things (Tarski did); I just mean I don't. The reason is practical: the usage I avoid can be confusing, particularly in discussions involving a concept of "truth preservation" as a condition of good argument.

${ }^{17}$ By "it" he here intends to refer to a future history, dismissing as a nonactual possibility a branching world such as Tree (p. 209).

${ }^{18}$ This is not true for another important stit concept, the "deliberative" stit, as defined first by von Kutschera 1986 and later (as reported in Belnap 1989) by J. Horty.

${ }^{19}$ See the discussion of the Ten minute mile in Belnap 1989.

${ }^{20}$ The words of the definiendum can regrettably suggest that $Q$ is settled true throughout 
$i$; perhaps there is a better phrase. To appreciate the concept behind the words, think of $i$ not as a set with members, but just as an unstructured instant or "time".

${ }^{21}$ Although it is sometimes useful to think of the upper cusp as a "point", it is well to bear in mind that the upper cusp need not be a complete past. In fact the most typical case may well be the one in which the upper cusp for $Q$ at $m$ is the set of all proper predecessors of $m$, which will certainly be incomplete unless $m$ participates in a violation of the Semi-lattice condition. For example, if the $Q$ in question is that $\mathrm{I}$ am at the restaurant at six, Murphy's Law suggests that it may well take right smack up to six in order to settle that fact in my favor; in which case the upper cusp is the entire set of proper predecessors of the moment of my being at the restaurant at six. Some people think this is a defect in the theory; I think it is a defect in the world.

${ }^{22}$ The definiendum contains the notation " $[\alpha$ stit: $Q]$ ", but that is just a communication device; I am not really defining a concept that depends on this notation. I use this device again in several upcoming definitions.

${ }^{23}$ This statement is too strong; when we come to worrying about the interaction of the progressive tenses with agency, these very same problems will come back in another and no less difficult form. Still, it is only fair to remember that the progressive tenses themselves are a matter of considerable difficulty, quite independent of agency.

\section{REFERENCES}

Aqvist, L.: 1974, 'A New Approach to the Logical Theory of Actions and Causality', in S. Stenlund (ed.), Logical Theory and Semantic Analysis, Essays Dedicated to Stig Kanger on his Fiftieth Birthday, D. Reidel, Dordrecht, pp. 73-91.

Aqvist, L.: 1978, 'An Analysis of Action Sentences Based on a 'Tree' System of Modal Tense Logic', in C. Rohrer (ed.), Papers on Tense, Aspect and Verb Classification, TBL Verlag Gunter Narr, Tubingen, pp. 111-61.

Belnap, N.: 1988, 'Declaratives Are Not Enough', Philosophical Studies 59, 1-30.

Belnap, N.: 1989, 'Backwards and Forwards in the Modal Logic of Agency', unpublished manuscript, University of Pittsburgh, Department of Philosophy, September, forthcoming in Philosophy and Phenomonological Research in early 1993.

Belnap, N. and Perloff, M.: 1989, 'Seeing to it That: a Canonical Form for Agentives', Theoria 54, 175-99. W. Edelberg pointed out to us that the informal semantic account of stit is garbled in this paper; the account is corrected in the version of this paper republished in H. E. Kyburg, Jr., R. P. Loui, and G. N. Carlson (eds.): 1990, Knowledge Representation and Defeasible Reasoning, Studies in Cognitive Systems, vol. 5, Kluwer Academic Publishers, Dordrecht, pp. 167-90.

Bressan, A.: 1972, A General Interpreted Modal Calculus, Yale University Press, New Haven.

Bressan, A.: 1972a, 'On the Usefulness of Modal Logic in Axiomatizations of Physics', in Schaffner and Cohen, pp. 285-304.

Bressan, A.: 1989, 'Comments on Gupta's 'Logic of Common Nouns', Unpublished manuscript, 1989.

Gabbay, D. and Guenthner, G. (ed.): 1984, Handbook of Philosophical Logic, Vol. II: Extensions of Classical Logic. Synthese Library, Studies in Epistemology, Vol. 165, D. Reidel Publishing Company, Dordrecht. 
Gupta, A.: 1980, The Logic of Common Nouns: An Investigation in Quantified Modal Logic. Yale University Press, New Haven.

Hamblin, C. L.: 1987, Imperatives. Basil Blackwell, Oxford and New York.

Kamp, J. A. W.: 1979, 'The Logic of Historical Necessity'. Unpublished manuscript.

Kripke, S.: 1959, 'A Completeness Theorem in Modal Logic', (abstract). The Journal of Symbolic Logic 24, 1-15.

Lewis, D.: 1986, On the Plurality of Worlds. Basil Blackwell, Oxford and New York.

McCall, S.: 1976, 'Objective Time Flow', Philosophy of Science 43, 337-62.

Mulloch, P.: 1988, 'Causing Harm: Criminal Law', Law and Philosophy 7, 67-105.

Plantinga, A.: 1974, The Nature of Necessity. Oxford University Press, Oxford.

Prior, A.: 1967, Past, Present, and Future. Oxford University Press, Oxford.

Prior, A.: 1968, Papers on Time and Tense. Oxford University Press, Oxford.

Schaffner, K. F. and Cohen, R. S. (ed.): 1972, PSA 1972. D. Reidel Publishing Company, Dordrecht.

Thomason, R. H.: 1970, 'Indeterminist Time and Truth-Value Gaps', Theoria 36, 26481.

Thomason, R. H.: 1984, 'Combinations of Tense and Modality'. In: Gabbay and Guenthner 1984, pp. 135-65.

Thomason, R. H. and Gupta, A.: 1980, 'A Theory of Conditionals in the Context of Branching Time', The Philosophical Review 89, 56-90.

Thomson, J.: 1977, Acts and Other Events, Cornell University Press, Ithaca.

von Kutschera, F.: 1986, 'Bewirken', Erkenntnis 24, 253-81.

von Neumann, J. and Morgenstern, O.: 1944, Theory of Games and Economic Behavior Princeton University Press, Princeton.

Zampieri, G.: 1982, 'Diffeomorphisms Constructively Associated with Mutually Diverging Spacetimes, which Allow a Natural Identification of Event Points in General Relativity', Atti Acc. Naz. Lincei (VIII) 73, 132-37, 221-25.

Zampieri, G.: 1982-83, 'A Choice of Global 4-Velocity Field in General Relativity', Atti Istituto Veneto Scienze, Lettere e Arti 141, 201-6.

Manuscript submitted Oct. 17, 1989

Department of Philosophy

University of Pittsburgh

Pittsburgh, PA 15260

U.S.A. 